\title{
STUDY ON TRANSFORMER FAULT DIAGNOSIS TECHNOLOGY OF VMD LOCAL SIGNAL DE-NOISING BASED ON KURTOSIS - APPROXIMATE ENTROPY
}

\author{
Dingke CHEN*, Changbin MAO, Bin QIN \\ State Grid Chongqing Beibei Power Supply Company, Chongqing 400700, China \\ * dingkchen@aliyun.com
}

\begin{abstract}
With the needs of social development, the scale of power equipment continues to expand. Among them, the transformer, as the core equipment in the power system, plays a key role in the safe and stable operation of the power system. However, in the field where the field strength is too high, partial breakdown of insulating media, that is the partial discharge occurs, which brings certain threats and damage to the safe operation of the power system. Therefore, this article uses the kurtosis-approximate entropy variational mode decomposition (VMD) partial discharge signal denoising method is used to preprocess the UHF partial discharge signal, through the simulation analysis and the result comparison, the feasibility of the method for denoising the partial signal of the transformer is clarified, designed to improve the safety and reliability of transformer operation.
\end{abstract}

Keywords: Transformer; kurtosis - approximate entropy; variational mode decomposition (VMD)

\section{INTRODUCTION}

As the scale of power equipment continues to expand, its safe and reliable operation is extremely important to the power system. Among them, the transformer is used as a hub type device in the power system. Once a fault occurs, it will have a serious negative impact on the power system, such as a major fire accident [5, 8]. In addition, during the transportation, installation and operation of the transformer, cracks, spurs, bubbles and floating metal particles may cause insulation defects on the reverse side due to various accidental factors, such insulation defects may be affected by the difference between the dielectric constant and the breakdown field strength, resulting in the occurrence of local breakdown, which is the common partial discharge phenomenon of the transformer $[6,10]$. However, partial discharge generates a series of gases, electric particles, and chemicals, which cause the temperature at the insulation defect to rise or corrosion, which deteriorates and damages the transformer insulator [7]. Over time, the entire power system will have serious problems or problems [2]. In summary, the insulation defect is the fuze of the system failure, and the partial discharge is the combustion improver. Therefore, real-time accurate monitoring of partial discharge types can quickly find the insulation defects of transformers, which is of great significance for effective diagnosis of transformer faults. In the on-line monitoring of power transformers, due to various factors in the field, there are many types of interference signals in the partial discharge signals monitored by the sensors, and it is difficult to obtain satisfactory noise cancellation effects only by hardware [3-4]. Even the detection device itself generates noise, such as noise caused by heat generated by components. Therefore, the acquisition of partial discharge signal for online monitoring systems requires further use of relevant digital signal processing techniques for interference suppression. In general, there are two main types of noise that interfere with on-site monitoring: first, white noise interference; second, periodic narrowband interference. Based on this, this paper uses the variational mode decomposition time-frequency analysis method to deal with the noise-causing signal, and uses the kurtosis-approximate entropy method to judge the interference component and eliminate it to provide positive power for the normal operation of the transformer.

\section{VARIATIONAL MODE DECOMPOSITION (VMD)}

In 2014, Konstantin Dragomiretskiy proposed an adaptive signal processing method,namely Variational Mode Decomposition (VMD). The method is capable of decomposing a complex signal $f$ into $K$ bandwidth-limited eigenmode components $u_{i}$ and a center frequency $\omega_{i}$, where, $i=1,2, \cdots K$. The constrained variational problem during VMD decomposition can be described by equation (1). 


$$
\begin{gathered}
\min _{\left\{u_{k}\right\},\left\{\omega_{k}\right\}}\left\{\sum\left\|\partial_{t}\left[\left(\sigma(t)+\frac{j}{\pi t}\right)^{*} u_{k}(t)\right] e^{-j \omega_{k} t}\right\|_{2}^{2}\right\} \\
\text { s.t. } \sum_{k} u_{k}(t)=f
\end{gathered}
$$

In the formula, the set of $K I M F$ components obtained by decomposition is represented by $\left\{u_{k}\right\}$, and $\left\{u_{k}\right\}=\left\{u_{1}, u_{2}, \cdots u_{k}\right\}$, the pulse function and the center frequency set of each component are represented by $\sigma(t)$ and $\left\{\omega_{k}\right\}$, respectively, where $\left\{\omega_{k}\right\}=\left\{\omega_{1}, \omega_{2}, \cdots \omega_{k}\right\}$ is. At this time, the Lagrangian multiplier $\lambda$ and the second penalty term $\alpha$ are introduced into it, and then converted into a nonconstrained problem, and the enhanced Lagrangian formula (2) is obtained. Then, the direct nonconstraint problem of the equation (2) is iteratively updated by means of the alternating direction multiplier method. And the corresponding center frequency $\omega_{i}$ and component $u_{i}$ are obtained by mathematical operations, where $i=1,2, \cdots, K$,which is represented by the equation (3) and the equation (4).

$$
\begin{aligned}
& L\left(\left\{u_{k}\right\},\left\{\omega_{k}\right\}, \lambda\right)= \\
& \alpha \sum\left\|\partial_{t}\left[\left(\sigma(t)+\frac{j}{\pi t}\right)^{*} u_{k}(t)\right] e^{-j \omega_{k} t}\right\|_{2}^{2} \\
& +\left\|f(t)-\sum_{k} u_{k}(t)\right\|_{2}^{2}+\left\langle\lambda(t), f(t)-\sum_{k} u_{k}(t)\right\rangle \\
& \qquad\left\langle\lambda(t), f(t)-\sum_{k} u_{k}(t)\right\rangle \text { is inner product }
\end{aligned}
$$
operation.

$$
\begin{array}{r}
\Lambda_{k}^{n+1}(\omega)=\frac{\Lambda(\omega)-\sum u_{i}(\omega)+\lambda(\omega) / 2}{1+2 \alpha\left(\omega-\omega_{k}\right)^{2}} \\
\omega_{k}^{n+1}=\frac{\int \omega\left|u_{k}(\omega)\right|^{2} d \omega}{\int_{0}^{\infty}\left|u_{k}(\omega)\right|^{\Lambda} d \omega}
\end{array}
$$

In the formula, the frequency is represented by $\omega$, and the Fourier transform of $f, u$, and $\lambda$ is represented by $\stackrel{\Lambda}{f}, \stackrel{\Lambda}{u}$, and $\lambda$ in turn.

\section{THE BASIC PRINCIPLE OF KURTOSIS- APPROXIMATE ENTROPY VMD}

\subsection{Kurtosis-approximate entropy model}

The kurtosis index $K$ is highly sensitive to the impact components in the signal. at the same time, the signal distribution characteristics can also be presented in a quantitative manner. Therefore, it is often used as a judgment indicator in the diagnosis of vibration signal fault diagnosis [1]. The corresponding definition is as shown in equation (5).

$$
K=\frac{E\left(x^{4}\right)}{E^{2}\left(x^{2}\right)}=\frac{\frac{1}{N} \sum_{i=2}^{N}\left(x_{i}-\bar{x}\right)^{4}}{\left[\frac{1}{N} \sum_{i=2}^{N}\left(x_{i}-\bar{x}\right)^{2}\right]^{2}}
$$

Where, the discrete signal sequence and signal length are represented by $x$ and $N$ respectively. For the approximate entropy, the complexity of the signal can also be described. If the corresponding value is non-negative, then the corresponding discrete event sequence complexity can be expressed in a quantitative manner. In addition, the complexity of the sequence is proportional to the size of the value, ie the larger the value, the more complex the corresponding sequence. The definition of kurtosisapproximate entropy is shown in equation (6).

$$
K_{A}=K * \text { Apen }
$$

\subsection{The basic idea of kurtosis-approximate entropy VMD}

When the input signal is a single impact, then the kurtosis index $K$ can achieve a better output. Due to the partial discharge inside the transformer, the corresponding signal propagation will exhibit the characteristics of damped oscillation. Therefore, the partial discharge signal after the output processing is completed may have multiple impacts, thereby suppressing the determination of the kurtosis index, resulting in the phenomenon of missed detection[9] . At the same time, the decreasing ratio of $\mathrm{k}$ is significantly lower than the increasing ratio of approximate entropy. Therefore, consider combining the two to construct a new kurtosis-approximate entropy feature coefficient as a new information measure. The basic idea of the algorithm is to use VMD to multi-modally decompose the signal and calculate the corresponding kurtosis-approximate entropy for each component obtained. When the obtained value exceeds the threshold range, then such a component can be regarded as an effective component of the Partial discharge signal, and an effective noise reduction signal is obtained by reconstruction.

\section{SIMULATION ANALYSIS OF VMD INTERFERENCE SUPPRESSION BASED ON KURTOSIS-APPROXIMATE ENTROPY}

\subsection{Only white noise interference}

The partial discharge signal is simulated by the single and double exponential decay oscillation mathematical model described above, and the white noise signal of $-3 \mathrm{~dB}$ is used as the superimposed 
signal ratio. The corresponding spectrum and white noise signal waveform are shown in Fig. 1.

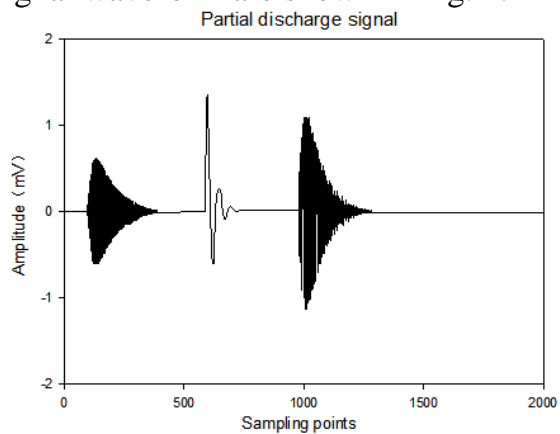

(a) original signal

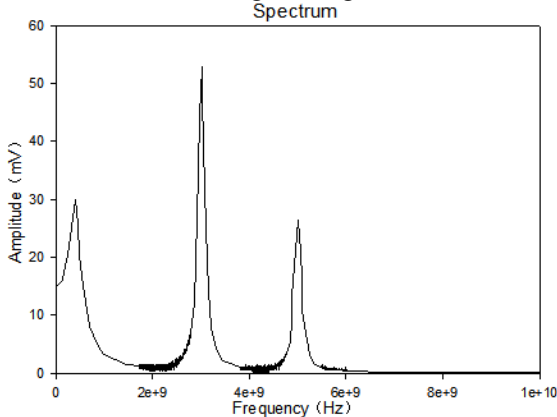

(b) The spectrum corresponding to the original signal

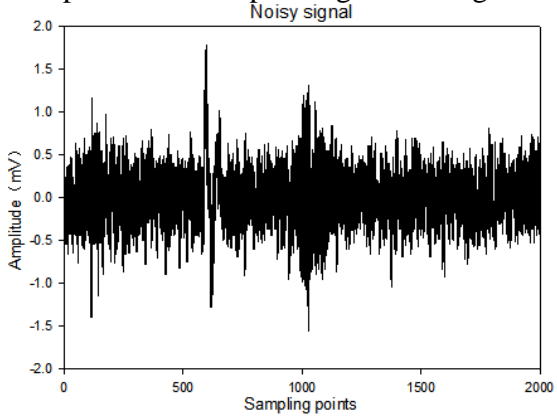

(c) White noise signal

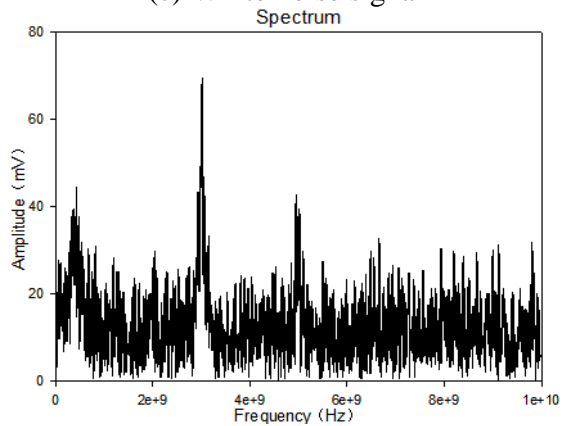

(d) Spectrum corresponding to white noise signal

Fig. 1. Raw and white noise partial discharge signals and corresponding spectra

The decomposition modal number is 10 , other VMD parameters are default values, and the kurtosisapproximate entropy threshold is set to 0 after standardization. After the kurtosis-approximate entropy VMD suppression interference algorithm is processed, the noise reduction signal and its spectrum, waveform and denoising error waveform are shown in Fig. 2.

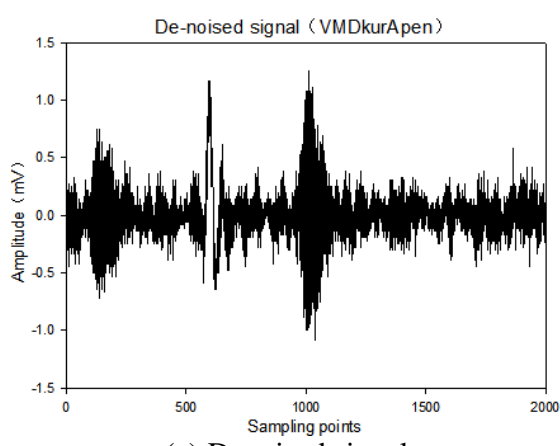

(a) Denoised signal

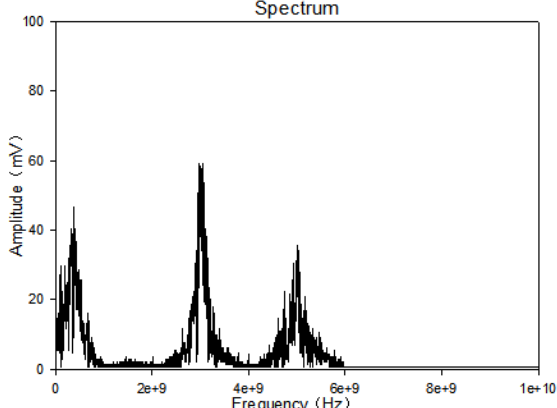

(b) Spectral corresponding to the signal after denoising

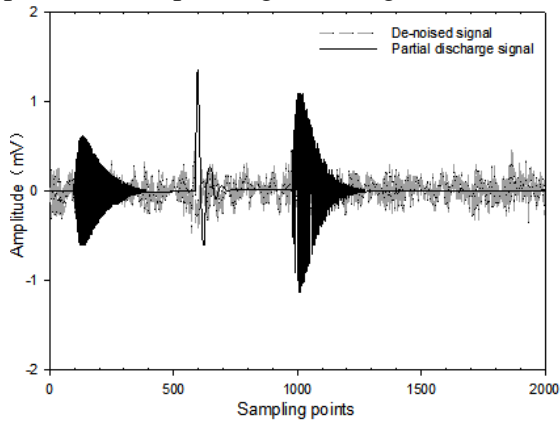

(c) Denoised signal and partial discharge signal waveform

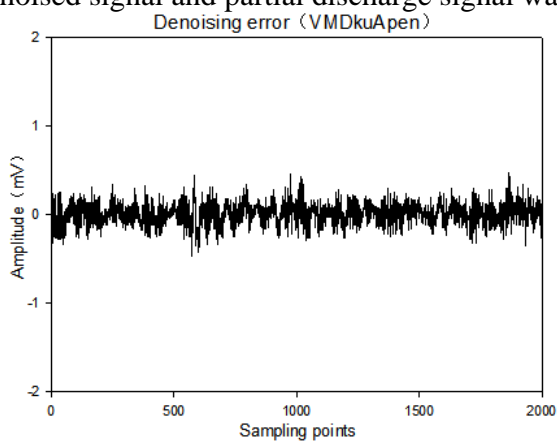

(d) Denoising error

Fig. 2. Signal, spectrum and denoising error after denoising by VMD algorithm

When superimposing a white noise signal with a signal-to-noise ratio of $\{-14 d B,-13 d B, \cdots 5 d B\}$, select the output signal-to-noise ratio (SNR), root mean square error (RMSE), signal distortion ratio (SDR) and noise suppression ratio (NRR) to evaluate the performance of the algorithm. The effect obtained after denoising is shown in Fig. 3. It can be seen that the VOD algorithm based on the kurtosisapproximate entropy method can effectively denoise in the background of different intensity white noise, and the running time of the algorithm is about $3.7 \mathrm{~s}$. 

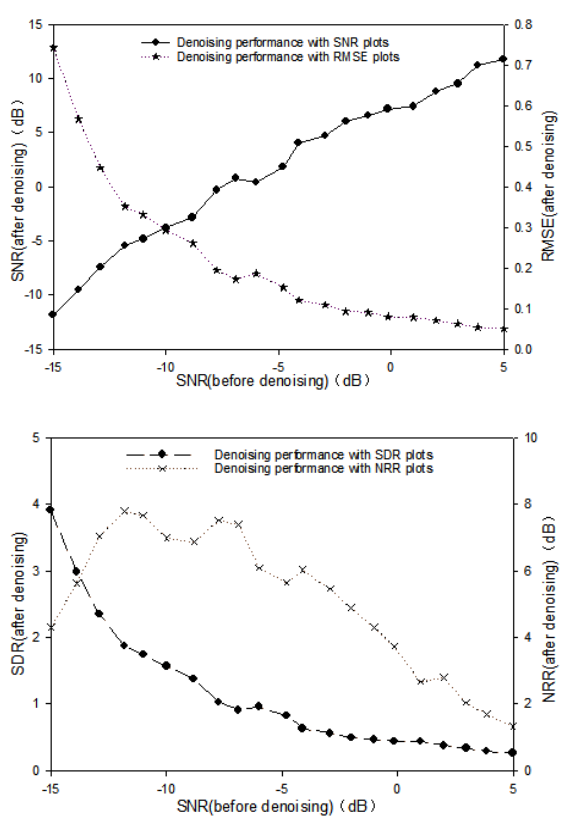

Fig. 3. VMD (kurApen) algorithm for noise cancellation (white noise) performance of SNR, RMSE, SDR and NRR

\subsection{Only periodic narrowband interference}

In the case where the remaining conditions are equivalent, the spectrum and the noise-diffused signal waveform when the superimposed signal ratio is $5.758 \mathrm{~dB}$ of periodic narrow-band interference is shown in Fig. 4. At the same time, after the kurtosisapproximation-based VMD suppression interference algorithm is processed, the obtained noise reduction signal and its spectrum and denoising error are shown in Fig. 5 .

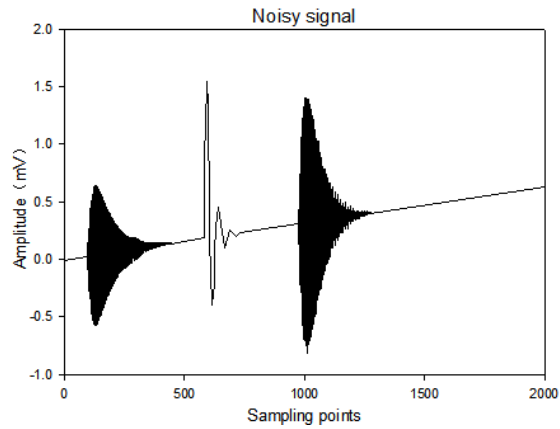

(a) Polluted noise signal

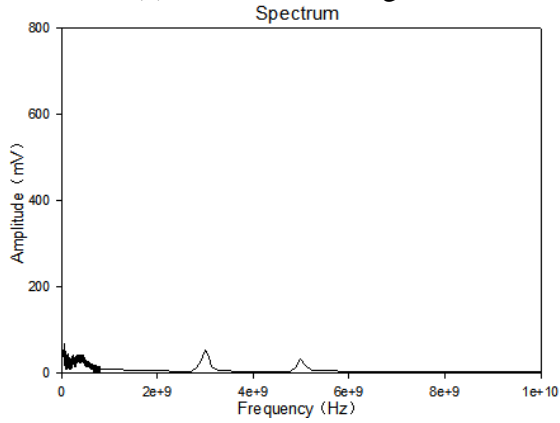

(b) Spectral corresponding to the polluted noise signal

Fig. 4. Noise signal and its spectrum

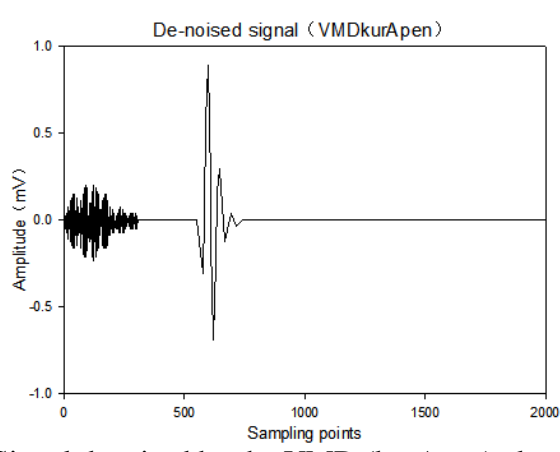

(a) Signal denoised by the VMD (kurApen) algorithm

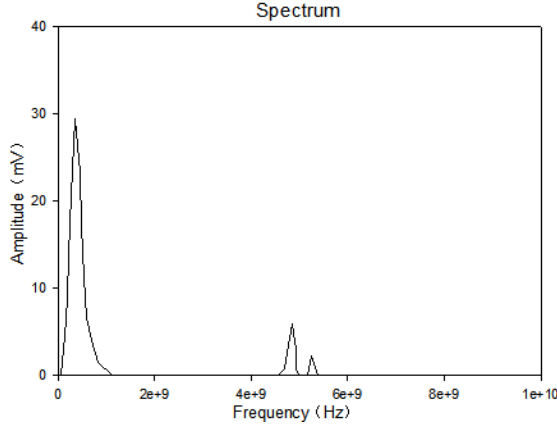

(b) Spectral corresponding to the signal after denoising

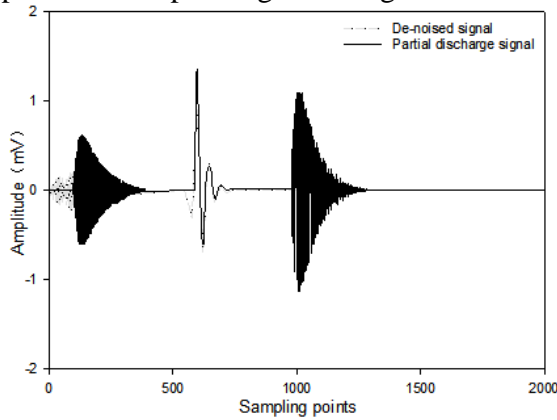

(c) Partial discharge signal and denoised signal waveform

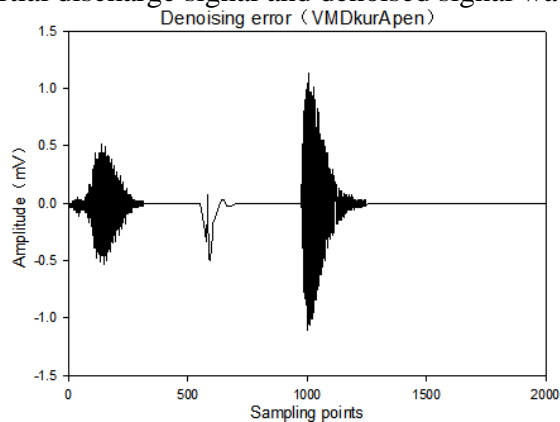

(d) Denoising error

Fig. 5. Denoising signal, corresponding spectrum and denoising error under VMD (kurApen) algorithm

The periodic narrow-band interference of 20 different signal-to-noise ratio is superimposed, and the obtained denoising effect is shown in Fig. 6. It can be seen that the VMD algorithm based on the kurtosis-approximate entropy method can achieve better denoising effect under the background of narrowband interference with different intensity periods. At the same time, the algorithm runs for about $12.7 \mathrm{~s}$. 


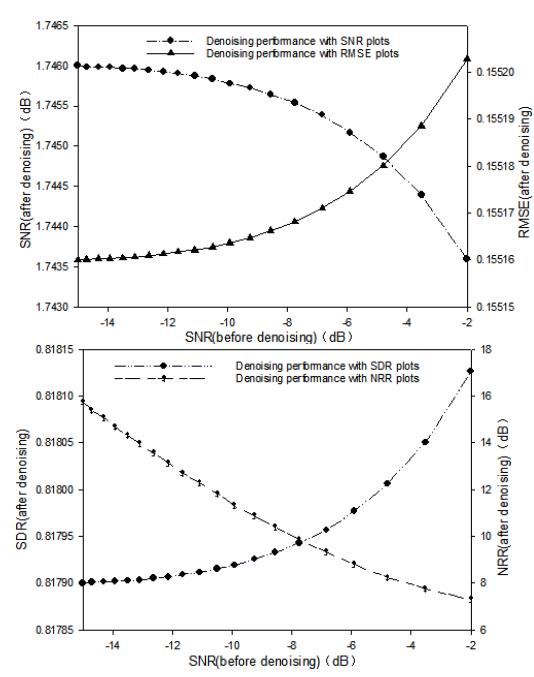

Fig. 6. VMD (kurApen) algorithm for noise reduction (narrowband interference) performance of SNR, RMSE, SDR and NRR

\subsection{White noise and periodic narrowband mixed interference}

The above-mentioned single and double exponential decay oscillation mathematical model is used to simulate the partial discharge signal, and the white noise signal with signal-to-noise ratio of $-3 \mathrm{~dB}$ and periodic narrow-band interference of $6.630 \mathrm{~dB}$ are superimposed to obtain the signals with noise waveform and its spectrum. Number of decomposition modes is 10 , and kurtosis approximate entropy threshold is set to 0 after standardization, and remaining parameters are set to default values. The signal spectrum, waveform and denoising error waveform obtained after the noise reduction processing by the VMD suppression interference algorithm with kurtosis-approximate entropy. The white noise signal with a signal-to-noise ratio of $\{-14 d B,-13 d B, \cdots, 5 d B\}$ the denoising effect under as shown algorithm based on the kurtosis-approximate entropy method can still achieve better denoising effect under different intensity white noise and periodic narrowband interference, and the algorithm running time is about $4.5 \mathrm{~s}$.

\section{MEASURED DATA PROCESSING OF VMD INTERFERENCE SUPPRESSION BASED ON KURTOSIS-APPROXIMATE ENTROPY}

Acquire an overclocked signal obtained by two sensing couplings simultaneously by means of an industrial computer installed near the transformer. Continuous monitoring of one $330 \mathrm{kv}$ transformer in one year, the system sampling rate is $1 \mathrm{GHz}$, the sampling event is $2 \mu \mathrm{s}$, and the continuous acquisition time interval is $2 \mathrm{~h}$. The data is transmitted in real time through the optical cable to the online monitoring server located in the main control room of the substation. The partial discharge electromagnetic signal monitored by the sensor in a certain insulation deterioration state is shown in Fig. 8(a). The superimposed signal ratio is $-3 \mathrm{~dB}$ white noise and $12.2308 \mathrm{~dB}$ periodic narrowband interference, and the spectrum and waveform of the white noise signal with a signal-to-noise ratio of $-3.8250 \mathrm{~dB}$ are obtained, as shown in Fig. 8. The number of decomposition modes and the entropy threshold at this time are 10 and 6.9 , respectively.

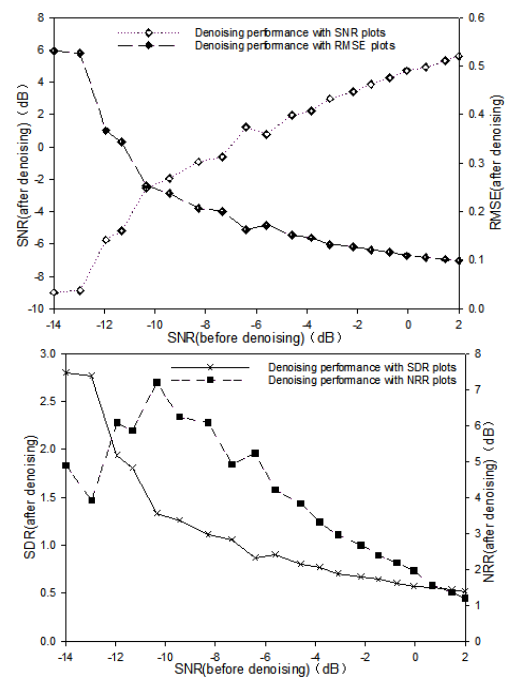

Fig. 7. VMD (kurApen) algorithm for noise cancellation (white noise and narrowband interference) performance of SNR, RMSE, SDR and NRR

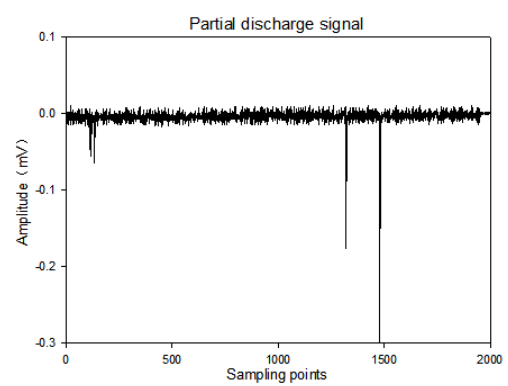

(a) Original signal

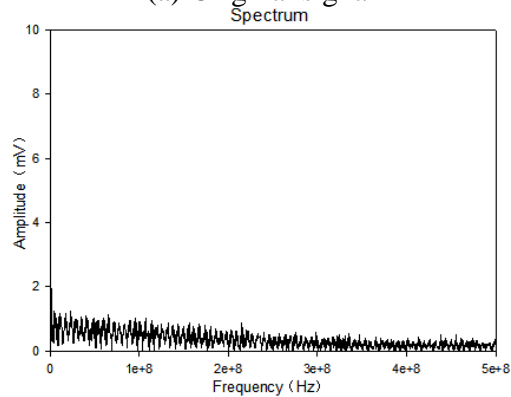

(b) The spectrum corresponding to the original signal

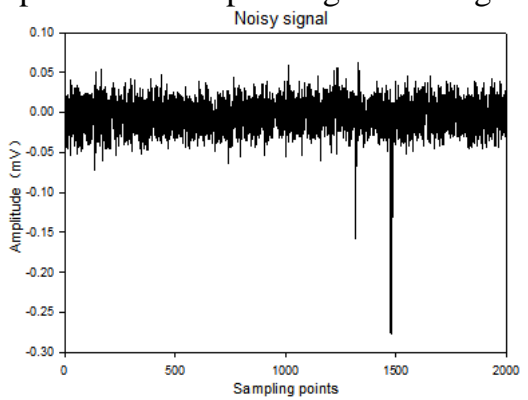

(c) Noisy signal 


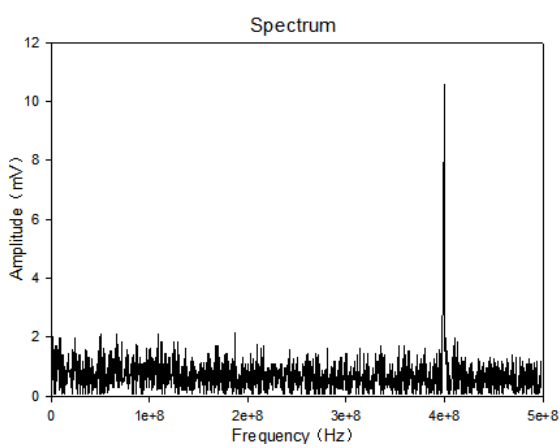

(d) Spectral corresponding to the noisy signal

Fig. 8. Measured raw and noise-induced (white noise and periodic narrow-band interference) partial discharge signals and their spectra

\section{MEASURED RESULTS COMPARISON}

In this paper, the traditional signal denoising method EDM is used to compare and verify the advantages of local signal denoising transformer fault diagnosis technology of VMD based on kurtosis and approximate entropy. The measured data and denoising errors of conventional ema and VMD (KurApen) after denoising are shown in figure 9. The denoising effects of EDM and VDM methods were compared and tested, and the results are shown in figure 10 .
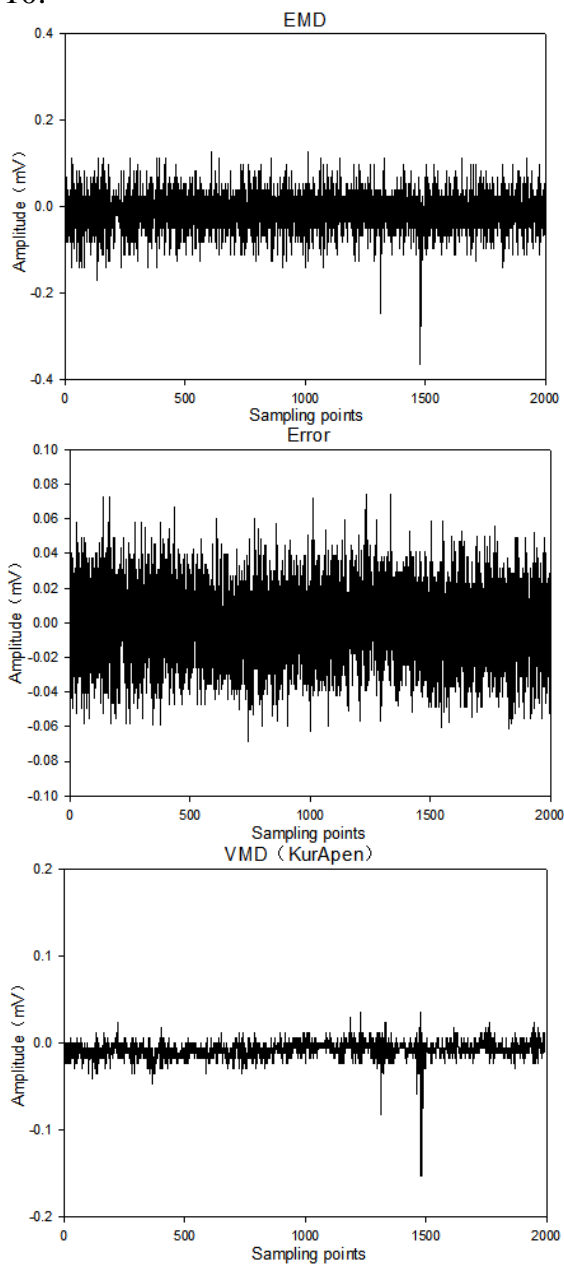

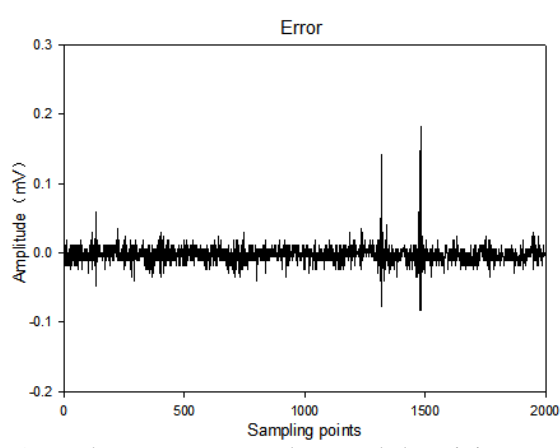

Fig. 9. Actual measurement data and denoising error after traditional ema and VMD (KurApen) denoising
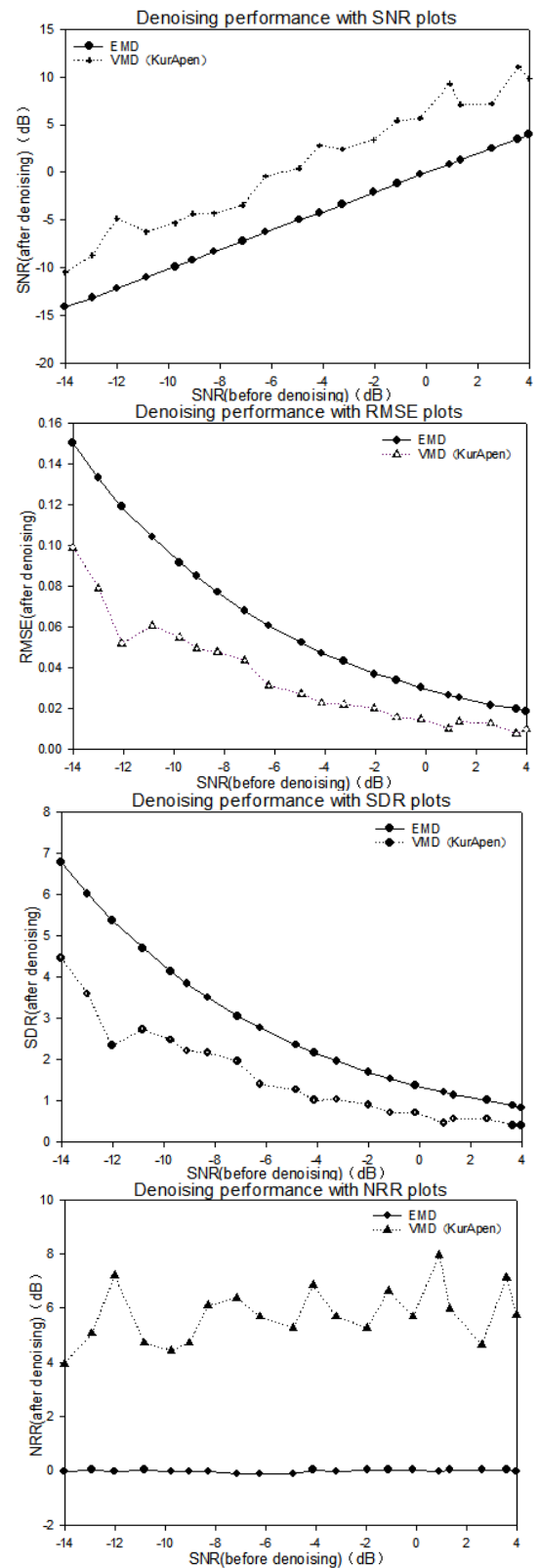

Fig. 10. EMD and VMD (KurApen) algorithm for noise cancellation (white noise and narrowband interference) performance of SNR, RMSE, SDR and NRR

It can be seen that the denoising effect of VMD (KurApen) under different indicators is better than that of traditional EMD, which confirms the feasibility and effectiveness of the denoising method of PD signal. Therefore, in the actual project, the 
VMD PD signal denoising method based on kurtosisapproximate entropy can be reasonably used to deal with white noise and narrowband interference, and the effect and efficiency of noise reduction can be improved.

\section{SUMMARY}

Under different types and different noise intensities, this paper proposes a VOD partial discharge signal denoising method based on kurtosisapproximate entropy. Through simulation analysis, the output signal-to-noise ratio (SNR), root mean square error (RMSE), signal distortion ratio (SDR) and noise rejection ratio (NRR) are selected to evaluate the performance of the algorithm. It is found that the kurtosis-approximate entropy-based VMD PD signal denoising method is superior to the traditional EMD and can achieve better denoising effect. Secondly, based on the analysis the measured data, the effectiveness of the denoising method based on the kurtosis-approximate entropy of the VMD PD signal denoising method is confirmed. Finally, for the measured PD signal, the VMD PD signal denoising algorithm based on kurtosis-approximate entropy can be selected for preprocessing.

\section{REFERENCES}

1. Chen XY. Research on gearbox vibration signal denoising and feature extraction method. Taiyuan University of Technology 2018.

2. Ding SY, Shen Q, Chen Y. Electrical diagnostic technology for partial discharge of power transformers. Science and Technology Outlook 2016; 26(33).

3. Dong J. Research and design of on-line monitoring system for partial discharge of power transformers. Transactions of Electrical Engineering 2017; (1): 2225.

4. Ge XL. Research on online monitoring and fault diagnosis method of transformer based on DGA. Anhui University of Science and Technology 2018.

5. Luo XL. Research on Fault Diagnosis Technology of Transformer. Engineering Technology: Abstracts 2016.

6. Mi XY, Su BG, Zhang GS, et al. Discussion on Judging Method of Partial Discharge of Transformer. Transformer 2018; (4): 66-67.
7. Sun ZQ. Research on Partial Discharge Detection Technology of Electrical Equipment. Electronic Production 2018; (2).

8. Wang B. Research on Causes and Treatment Methods of Transformer Faults in Power System. Science and Technology Innovation and Application 2017; (34): 81-81.

9. Wang LL, Liu HL, Gao SG. Simulation Study on Propagation Characteristics of Partial Discharge Pulse Current in UHV Transformer. High Voltage Apparatus 2018; 54(11): 227-232.

10. Yan SY. Causes and Control Measures for Partial Discharge of Transformers. 2017 Annual Meeting of the Electrification Committee of China Railway Society and New Technology Seminar 2017.

Received 2019-11-07

Accepted 2020-02-09

Available online 2020-02-17

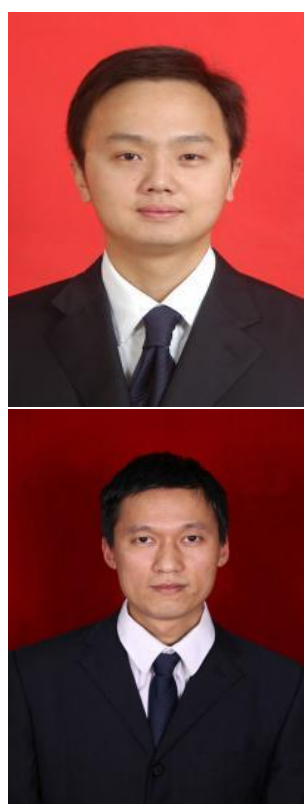

Dingke CHENG, born in 1982, gained the bachelor's degree from North China Electric Power University in 2006. Now he is an engineer and works in State Grid Chongqing Beibei Power Supply Company, China. He is interested in high voltage technology.

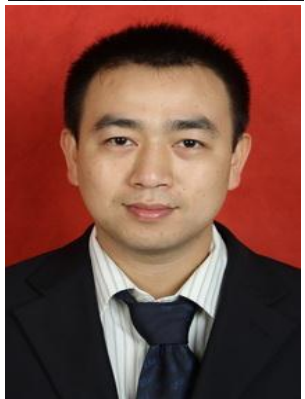

Changbin MAO, born in 1973, gained the bachelor's degree from Internet College of Sichuan Uuniversity in 2014. He is now an senior engineer and works in State Grid Chongqing Beibei Power Supply Company, China. He is interested in electrical engineering $\&$ its automation.

Bin QIN, born in 1978, gained the master's degree from Hunan University, China. He is now an engineer and works in State Grid Chongqing Beibei Power Supply Company, China. He is interested in high voltage 